\title{
Proof systems for Geometric theories (PROGEO)
}

\author{
Elaine Pimentel ${ }^{1}$
}

\author{
${ }^{1}$ Departamento de Matemática \\ Universidade Federal do Rio Grande do Norte
}

\begin{abstract}
We plan to study the problem of finding conservative extensions of first order logics. In this project we intend to establish a systematic procedure for adding geometric theories in both intuitionistic and classical logics, as well as to extend this procedure to bipolar axioms, a generalization of the set of geometric axioms. This way, we obtain proof systems for several mathematical theories, such as lattices, algebra and projective geometry, being able to reason about such theories using automated deduction.
\end{abstract}

Resumo. Neste projeto de pesquisa, pretendemos estudar o problema de estender, conservativamente, lógicas de primeira-ordem. Pretendemos estabelecer um procedimento sistemático para adicionar teorias geométricas e extensões em lógicas clássica e intuicionista. Dessa forma, obtemos sistemas de provas para diversas teorias matemáticas, tais como reticulados, álgebra e geometria projetiva, provendo sistemas automáticos de dedução para tais teorias.

\section{Introduction}

One of the advantages of using sequent systems as a frameworks for logical reasoning is that the resulting calculi are often simple, have good proof theoretical properties (like cut-elimination, consistency, etc) and can be easily implemented, e.g., using rewriting.

Hence it would be heaven if we could add axioms in mathematical theories to first order logics and reason about them using all the machinery already built for the sequent framework. Indeed, the general problem of extending standard prooftheoretical results obtained for pure logic to certain class of non-logical axioms has been focus of attention for quite some time now.

The main obstacle for this agenda is that adding non-logical axioms to systems while still maintaining the good proof theoretical properties it is not an easy task. In fact, as described in Negri and von Plato 1998, if $A, B$ are atoms and the axioms $\vdash A \supset B$ and $\vdash A$ are added to the sequent system $L J$ for intuitionistic logic Gentzen 1935a], then the sequent $\vdash B$ can be derived using cut:

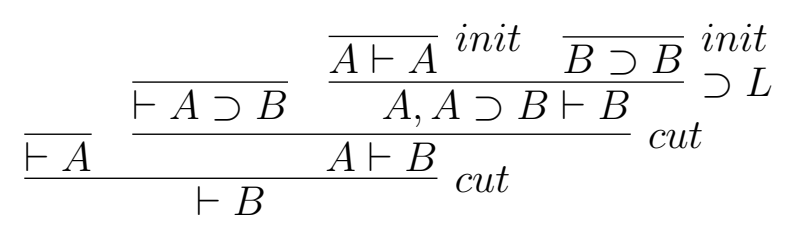

But it is easy to see that there is no proof of this sequent without cut. That is, the resulting system is not cut-free: applications of the rule cut can not be eliminated. 
One way of circumventing this problem is by treating axioms as theories, added to the sequent context. This is already in Gentzen's consistency proof of elementary arithmetic in Gentzen 1935b. Now the derivations have only logical axioms as premisses, and cut elimination applies. In the example above, we can derive $B$ from $A, A \supset B$ without a problem

$$
\frac{\overline{A \vdash A} \text { init } \overline{A, B \vdash B}}{A, A \supset B \vdash B} \supset L
$$

But we can do better by transforming the axioms above into inference rules. In fact, if $A, B$ are atomic formulas and $C$ an arbitrary formula then, in the presence of $A \supset B$, if $B$ proves $C$ then $A$ also proves $C$. On the other hand, in the presence of $A$, if $A$ proves $C$, then $C$ is provable (the $A$ is irrelevant since it is already there). This induces the inference rules

$$
\frac{\Gamma, B \vdash C}{\Gamma, A \vdash C} A \supset B \quad \frac{\Gamma, A \vdash C}{\Gamma \vdash C} A
$$

The sequent $\vdash B$ now has the (cut-fre) proof

$$
\begin{aligned}
& \frac{\overline{B \vdash B}^{\text {init }}}{\frac{1}{\vdash B}^{A}} \stackrel{A}{ } \mathrm{D} B
\end{aligned}
$$

In this project, we intend to propose a systematic way of adding inference rules to sequent systems. The proposal will be based on the notions of focusing and polarities, illustrated next.

\section{A gentle introduction to polarities and focusing}

We will start by generalizing the example above. Let $B$ be a formula and $\Gamma$ be a multiset of formulas. Consider attempting to build a proof of the following two-sided sequent

$$
\Gamma, A_{1} \supset \cdots \supset A_{n} \supset A_{0} \vdash B,
$$

in which the distinguished implication is such that $n \geq 1$ and $A_{0}, \ldots, A_{n}$ are atomic formulas. In general, there are many ways to proceed with attempting to build a cut-free proof of this sequent and we characterize them as one of the following four possibilities. This sequent can be the conclusion of

1. a structural rule (weakening or contraction) or the initial rule;

2. a right introduction rule, if $B$ is not an atomic formula;

3. a left-introduction rule that introduces a formula in $\Gamma$; or

4. the implication-introduction rule that introduces the distinguished implication.

The number of possible choices here could be large, particularly if $\Gamma$ contains a large number of formulas. If we chose the fourth of these possibilities, the proof would look as follows (at least in the intuitionistic setting):

$$
\frac{\Gamma \vdash A_{1} \quad \Gamma, A_{2} \supset \cdots \supset A_{n} \supset A_{0} \vdash B}{\Gamma, A_{1} \supset \cdots \supset A_{n} \supset A_{0} \vdash B} L \supset
$$


Note that we again have a large number of possible ways to proceed in attempting to prove the right premise: indeed, if $n \geq 2$, we have all the same choices as before. Clearly, those choices - and their multiplicative effects as we search for a sequence of inference steps that terminates in a proof - are in desperate need of being structured somehow. Focused proof systems provide such structure using the following two devices.

Focused rule application If you chose to apply the implication-left introduction on the distinguished implication, then you also commit to repeat the implication-left rule on the right premise until the atomic formula $A_{0}$ results. That is, the left-introduction applied to the distinguished implication results in the following derived inference rule

$$
\frac{\Gamma \vdash A_{1} \quad \ldots \quad \Gamma \vdash A_{n} \quad \Gamma, A_{0} \vdash B}{\Gamma, A_{1} \supset \cdots \supset A_{n} \supset A_{0} \vdash B} L \supset \quad n \text { times. }
$$

Polarization Although the focused application of inference rules provides structure to attempts to build proofs, there are still so many remaining choices, that it is possible to impose two different "protocols" for restricting choices further. The $Q$-protocol insists that the first $n$ premises above are trivial, meaning that they are proved by the initial rule. Following that protocol, we have $A_{i} \in \Gamma$ for $1 \leq i \leq n$. Thus, if we set $\Gamma^{\prime}$ to be the result of removing all occurrences of $A_{1}, \ldots, A_{n}$ from $\Gamma$, then the derived inference rule above becomes

$$
\frac{\Gamma^{\prime}, A_{1}, \ldots, A_{n}, A_{0} \vdash B}{\Gamma^{\prime}, A_{1}, \ldots, A_{n}, A_{1} \supset \cdots \supset A_{n} \supset A_{0} \vdash B} .
$$

The second protocol, the $T$-protocol insists that the right-most premise is trivial: that is, $A_{0}$ and $B$ are the same atomic formula. Thus, the derived inference rule above becomes

$$
\frac{\Gamma \vdash A_{1} \quad \ldots \quad \Gamma \vdash A_{n}}{\Gamma, A_{1} \supset \cdots \supset A_{n} \supset A_{0} \vdash A_{0}} .
$$

Using the $Q$-protocol, the proof-search semantics of the implication $A_{1} \supset$ $\ldots \supset A_{n} \supset A_{0}$ is given by forward-chaining: if you have assumptions $A_{1}, \ldots, A_{n}$ then you can add the assumption $A_{0}$. Using the $T$-protocol, the proof-search semantics of the same implication is given by back-chaining: in order to prove the conclusion $A_{0}$, attempt instead to prove each of $A_{1}, \ldots, A_{n}$. The names for the $Q$ and $T$ protocols comes from Danos, Joinet, and Schellinx [Danos et al. 1995]: in the $Q$ protocol, the tail ("queue") of an implication yields a trivial premise while in the $T$ protocol, the head ("tête") of an implication yields a trivial premise.

A more modern and flexible presentation of the $Q$ and $T$ protocols speaks, instead, of the polarity of formulas: for this example, the polarity given to atomic formulas is the most relevant. In particular, if all atomic formulas have a positive polarity, the $Q$-protocol is enforced, while if all atomic formulas have a negative polarity, the $T$-protocol is enforced.

The base systems we will consider in this project are the focused proof systems for classical and intuitionistic logics, $L K F$ and $L J F$, respectively Liang and Miller 2007. 
Liang and Miller 2009. Those systems extend both the notion of focusing and polarity to all formulas, moving beyond the example above involving only implications and atomic formulas. In particular, focused rule applications imply that focus is transferred from conclusion to premises in derivations. This process goes on until either the focused phase ends (depending on the polarity of the focused formula), or the derivation ends. Once the focus is released, the formula is eagerly decomposed into subformulas, which are ultimately stored in the context.

Reading derivations from the root upwards, this forces a sequent derivation to be organized into focused phases, each of them corresponding to an application of a synthetic inference rule [Chaudhuri 2008], where the focused formula is rewritten into (some of) its subformulas.

There is a class of formulas corresponding to particularly interesting synthetic rules: the bipolars. Bipolars are formulas in which polarity can change at most once among its subformulas. This means that focusing on a bipolar $A$ gives rise to (possibly many) synthetic inference rules having simple shape, with leaves involving only atomic subformulas of $A$. We call a synthetic inference rule corresponding to the bipolar $A$ a bipole for $A$.

In this project, we will present a careful study of bipoles, giving a fresh view to an old problem: how to incorporate inference rules encoding axioms into proof systems for classical and intuitionistic logics.

A key step in transforming a formula into synthetic inference rules involves attaching a polarity to atomic formulas and to some logical connectives. Since there are different choices for assigning polarities, it is possible to produce different synthetic inference rules for the same (unpolarized) formula. In the example above, there are (at most) $2^{n+1}$ different possible polarizations for the atomic formulas in $A_{1} \supset \cdots \supset A_{n} \supset A_{0}$, each of them corresponding to a different bipole.

We show that this flexibility allows for the generalization of different approaches for transforming axioms into sequent rules present in the literature (more notably the series of works Negri 2003, Negri and von Plato 2011, Negri 2016 and Viganò 2000).

\section{A case study: geometric axioms}

The main challenge in this effort is to determine a general procedure that guarantees that such extensions preserve good proof-theoretical properties.

A remarkable step in that direction was the careful investigation of geometric axioms. Geometric axioms are first-order formulas that can be converted into (natural deduction/sequent) inference rules having "a certain simple form in which only atomic formulas play a critical part", as described by Simpson Simpson 1994. And this "simple rules for atomic formulas" motto seems to be the core of success in this endurance in the approaches/extensions present in the literature [Dyckhoff and Negri 2015]. In this work, we come back to the inception of the axioms-as-rules problem, showing that the combination of bipolars and focusing is the real essence of "simple rules for atomic formulas".

There are many examples of geometric theories in different areas of logic and 
mathematics, such as geometry, algebra, topology and category theory (see some examples in Section 5).

We will illustrate next how to translate this class of axioms into synthetic inference rules.

Definition 1 A geometric implication is a first-order formula having the form

$$
\forall \bar{z}\left(P_{1} \wedge \ldots \wedge P_{m} \supset \exists \bar{x}_{1} M_{1} \vee \ldots \vee \exists \bar{x}_{n} M_{n}\right)
$$

where each $P_{i}$ is an atomic formula, each $M_{j}$ is a conjunction of atomic formulas $Q_{j_{1}}, \ldots, Q_{j_{k_{j}}}$, and none of the variables in the lists $\bar{x}_{1}, \ldots, \bar{x}_{n}$ are free in $P_{i} . A$ geometric theory is a finite set of geometric implications. We shall also assume that if the list of variables $\bar{x}_{i}$ is empty then $M_{i}$ is just an atom: otherwise, this formula can be written as a conjunction of geometric implications.

An example of a geometric implication is the transitivity axiom, stating that, for a binary relation $R$, if $x$ is related to $y$ and $y$ is related to $z$ then $x$ is related to $z$

$$
4=\forall x, y, z \cdot(R(x, y) \wedge R(y, z)) \supset R(x, z)
$$

Now, for polarizing this formula in $L K F$ or $L J F$, we can give to the atomic formula $R$ and the conjunction a positive or a negative polarity (the quantifiers and implication are neutral in $L K F / L J F)$. We then obtain the following four poralized formulas (bipolars)

$$
\forall x, y, z \cdot\left(R(x, y)^{ \pm} \wedge^{ \pm} R(y, z)^{ \pm}\right) \supset R(x, z)^{ \pm}
$$

As one can expect, different polarizations can give rise to different bipoles (inference rules). For this example, focusing on each and all these formulas in LJF (it holds also for $L K F$ ) will produce the following two inference rules (bipoles) ${ }^{1}$

$$
\frac{R(x, z), \Gamma \vdash C}{R(x, y), R(y, z), \Gamma \vdash C} 4_{G R S} \quad \frac{\Gamma \vdash R(x, y) \quad \Gamma \vdash R(y, z)}{\Gamma \vdash R(x, z)} 4_{R R}
$$

The rule $4_{G R S}$ appears in Negri 2005 and corresponds to backward-chaining, while the rule $4_{R R}$ is the transitivity rule studied in Viganò 2000, corresponding to forward-chaining. This implies that these works are different faces of the same coin, the latter being minted from focusing and polarization.

Moreover, we address these issues with a uniform presentation in both classical and intuitionistic first-order logics.

\section{Beyond geometric axioms}

It turns out that the set of bipolar formulas is strictly greater than the set of geometric formulas. As an example, in set theory, the following implication relates the subset and membership predicates

$$
\forall y z .(\forall x(x \in y \supset x \in z) \supset y \subseteq z) .
$$

\footnotetext{
${ }^{1}$ For details about the systems $L K F$ and $L J F$, as well as the process of transforming axioms to synthetic rules using focusing, please refer to [Marin et al. 2020].
} 
This formula yields a bipolar (but not geometric) formula in both $L K F$ and $L J F$ under any polarization of the binary atomic predicates $\in$ and $\subseteq$. Assuming that these predicates are given positive polarity, the corresponding $L J F$-synthetic inference rule is

$$
\frac{x \in y, \Gamma \vdash x \in z \quad y \subseteq z, \Gamma \vdash E}{\Gamma \vdash E} .
$$

Assuming that these predicates are given negative polarity, the corresponding LJFsynthetic inference rule is

$$
\frac{x \in y, \Gamma \vdash x \in z}{\Gamma \vdash y \subseteq z} .
$$

In both of these synthetic inference rules, $x$ is an eigenvariable for that rule.

This means that our bipoles/focusing method generalizes and goes beyond the ones present in the literature. In fact, it classifies all and only axioms that can be transformed to sequent rules.

The sole responsible for this is the fact that, in bipolars, the polarity can change at most once among its subformulas. This means that focusing on a bipolar will completely decompose such formula until getting to atoms, which will be either stored in the context (in this case it appears in premises), or will be principal in the initial axiom (in which case it appears in the conclusion). In this sense, the rules corresponding to the bipolars - the bipoles - can be seen as introduction rules for atoms.

\section{Examples in Mathematics}

We finish this study proposal by enumerating some examples of axioms in mathematics that can be analyzed within this project. Some of them appear in the book Negri and von Plato 2011, some are unpublished.

1. Partial order. Assuming the domain $\mathcal{D}=\{a, b, c \ldots\}$ and a binary relation $\leq$ in $\mathcal{D}$, we say that $\leq$ is a partial order over $\mathcal{D}$ if:

PO1 $\forall a . a \leq a$ (reflexivity).

PO2 $\forall a, b .(a \leq b) \wedge(b \leq a) \supset a=b$ (anti-simety).

PO3 $\forall a, b .(a \leq b) \wedge(b \leq c) \supset a \leq c$ (transitivity).

2. Strict partial order. Assuming the domain $\mathcal{D}=\{a, b, c \ldots\}$ and a binary relation $<$ in $\mathcal{D}$, we say that $<$ is a strict partial order over $\mathcal{D}$ if it satisfies transitivity and:

PO4 $\forall a . \neg(a<a)$ (irreflexivity).

It is easy to see that, if a relation $<$ satisfies [PO3] and [PO4], then it also satisfies:

PO5 $\forall a, b .(a<b) \supset \neg(b<a)$.

3. Projective geometry. An axiomatization of projective geometry starts by defining the basic domain and relations. Denoting points by $a, b, c, \ldots$ and lines by $l, m, n, \ldots$, we have that the basic relations are $a=b, l=m$ and $a \in l$. We will consider then the reflexivity and transitivity described before together with:

ER1 $\forall a, b .(a=b) \supset(b=a)$ (simmetry). 
4. Normal modal logic. Extensions of modal logic $K$ are determined by adding relational axioms to the original system. We describe some bellow.

$\mathrm{E} \forall w, o, r .(w R o \wedge w R r) \supset o R r$ (euclideanity)

D $\forall w . \exists o . w R o$ (seriality)

5. Torsion abelian groups. The main axiom of torsion abelian groups, that says that all objects has finite order, can be described as

$$
\forall x . \top \supset \bigvee_{n=1}^{\infty} n x=0
$$

6. Local rings. The main axiom of torsion abelian groups, that says that there exists exactly one maximal ideal, can be described as

$$
\forall x . \top \supset(\exists y .(x y=1)) \vee(\exists y \cdot(1-x) y=1))
$$

7. Set theory. There are many axioms in set theory falling into the bipolar setting. As an example, the following implication relates the subset and membership predicates:

$$
\forall y z .(\forall x(x \in y \supset x \in z) \supset y \subseteq z)
$$

\section{Conclusion}

We have illustrated how the notion of synthetic inference rule that is provided by sequent calculus notions of polarization and focusing can be used to provide inference rules that capture certain classes of axioms.

In particular, focused proof systems naturally lead to the notion of bipolar formulas and these result in synthetic inference rules that only need to mention atomic formulas.

We show that geometric formulas are examples of such bipolar formulas and that polarized versions of such formulas yield known inference systems derived from geometric formulas. Certain subsets of geometric formulas admit more than one polarization and these variations explain the forward-chaining and backwardchaining variants of their synthetic inference rules. Additionally, all of these results work equally well in both classical and intuitionistic logics using the corresponding $L K F$ and $L J F$ focused proof systems.

With this project, we plan to develop the application of such a framework of focusing and bipoles to mathematical theories.

This project will be in collaboration with:

- Dale Miller (École Polytechnique, France)

- Sonia Marin (University College London, UK)

- Carlos Olarte (UFRN, Brazil)

- Luiz Carlos Pereira (PUC-Rio, Brazil)

- Emerson Sales (UFRN, Brazil)

- Brunna Karla de Morais Souza Assunção (UFRN, Brazil)

- Warlike Richard da Silva Soares (UFRN, Brazil)

- Luana Mayara Lucas Leite (UFRN, Brazil)

- Francisco Jonatã Chaves de Lima (UFRN, Brazil)

- Cristiano Victor Medeiros da Silva (UFRN, Brazil) 


\section{References}

Chaudhuri, K. (2008). Focusing strategies in the sequent calculus of synthetic connectives. In Cervesato, I., Veith, H., and Voronkov, A., editors, Logic for Programming, Artificial Intelligence, and Reasoning, 15th International Conference, LPAR 2008, Doha, Qatar, November 22-27, 2008. Proceedings, volume 5330 of Lecture Notes in Computer Science, pages 467-481. Springer.

Danos, V., Joinet, J.-B., and Schellinx, H. (1995). LKT and LKQ: sequent calculi for second order logic based upon dual linear decompositions of classical implication. In Girard, J.-Y., Lafont, Y., and Regnier, L., editors, Advances in Linear Logic, number 222 in London Mathematical Society Lecture Note Series, pages 211-224. Cambridge University Press.

Dyckhoff, R. and Negri, S. (2015). Geometrisation of first-order logic. The Bulletin of Symbolic Logic, 21(2):123-163.

Gentzen, G. (1935a). Investigations into logical deduction. In Szabo, M. E., editor, The Collected Papers of Gerhard Gentzen, pages 68-131. North-Holland, Amsterdam.

Gentzen, G. (1935b). Untersuchungen über das logische schliessen. Mathematische Zeitschrift, 39:176-210.

Liang, C. and Miller, D. (2007). Focusing and polarization in intuitionistic logic. In Duparc, J. and Henzinger, T. A., editors, CSL 200\%: Computer Science Logic, volume 4646 of $L N C S$, pages 451-465. Springer.

Liang, C. and Miller, D. (2009). Focusing and polarization in linear, intuitionistic, and classical logics. Theor. Comput. Sci., 410(46):4747-4768.

Marin, S., Miller, D., Pimentel, E., and Volpe, M. (2020). From axioms to synthetic inference rules via focusing. Available at https://drive.google.com/file/d/ 1_gNtKjvmxyH7T7VwpUD0QZtXARei8t5K/view.

Negri, S. (2003). Contraction-free sequent calculi for geometric theories with an application to Barr's theorem. Arch. Math. Log., 42(4):389-401.

Negri, S. (2005). Proof analysis in modal logic. J. Philosophical Logic, 34(5-6):507544 .

Negri, S. (2016). Proof analysis beyond geometric theories: from rule systems to systems of rules. J. Log. Comput., 26(2):513-537.

Negri, S. and von Plato, J. (1998). Cut elimination in the presence of axioms. Bulletin of Symbolic Logic, 4(4):418-435.

Negri, S. and von Plato, J. (2011). Proof Analysis - a contribution to Hilbert's last problem. Cambridge University Press.

Simpson, A. K. (1994). The Proof Theory and Semantics of Intuitionistic Modal Logic. PhD thesis, College of Science and Engineering, School of Informatics, University of Edinburgh.

Viganò, L. (2000). Labelled Non-Classical Logics. Kluwer Academic Publishers. 